\section{Metalliferous content of drink- ing water and sediments in storage tanks of some schools in Erbil city, Iraq}

\author{
Hawraz Sami Khalid, ${ }^{1}$ Hoshyar Saadi \\ Ali, ${ }^{2}$ Dhary Alewy Almashhadany ${ }^{2}$ \\ ${ }^{1}$ Salahaddin University-Erbil, College of \\ Education, Department of Chemistry, \\ Erbil City, Kurdistan Region; \\ ${ }^{2}$ Department of Medical Lab Science, \\ College of Science, Knowledge \\ University, Erbil City, Kurdistan \\ Region, Iraq
}

\begin{abstract}
The present study was conducted to evaluate the quality of drinking water in randomly selected schools in Erbil city, Kurdistan Region, Iraq. The water quality indices such as the Heavy metal Pollution Index (HPI) and Heavy metal Evaluation Index (HEI) were applied to characterize water quality. Eighteen schools were incorporated and sampled for their water storage tanks available to students. Water samples and sediment samples from tanks floor were analyzed by Inductively Coupled Plasma Optical Emission Spectrometer for the determination of twenty-two metal elements. In drinking water samples, all detected metals did not exceed the permissible limits of the World Health Organization. The results of this study showed that the average values of HPI and $\mathrm{HEI}$ for $\mathrm{As}, \mathrm{Cd}, \mathrm{Cr}, \mathrm{Cu}, \mathrm{Fe}, \mathrm{Pb}, \mathrm{Mn}, \mathrm{Ni}$, and $\mathrm{Zn}$ were 54.442 and 0.221 , respectively. According to data of the water quality indices, the schools drinking water quality are good and suitable for drinking in terms of heavy metals. However, sediments samples contained high concentrations of all elements including the toxic heavy metals (As, $\mathrm{Cd}, \mathrm{Cr}$, and $\mathrm{Pb}$ ). Re-suspension of sediments into water column after refilling storage tanks can pose a serious threat to students drinking water from such vessels. It is therefore recommended that proper storage tanks are provided to the schools accompanied by continuous sanitation and hygiene practice to mitigate the corrosion of tanks to avoid health risks of toxic metals.
\end{abstract}

\section{Introduction}

The problem of water contamination with heavy metals is a worldwide challenge especially in developing countries where disposal of various agro-industrial wastes is less regulated by relevant authorities (Baldwin et al., 2016; Chowdhury et al., 2016; Ferronato and Torretta, 2019). More than thirty metals are known to pose threats to human health due to their low rate of excretion. More than 20 of these metals are defined as heavy metals (Duffus, 2002). Heavy metals are defined as elements with density $>5 \mathrm{~g} / \mathrm{cm}^{3}$ (Ali and Khan, 2018). Some metals are required for normal physiology of the human body but in low quantities. For instance, trace elements such as iron $(\mathrm{Fe})$, manganese $(\mathrm{Mn})$, magnesium $(\mathrm{Mg})$, zinc $(\mathrm{Zn})$, and sodium $(\mathrm{Na})$ are important cofactors for numerous enzymes and normal physiology of cells. In contrast, other metals are toxic even in low concentrations such as arsenic (As), lead $(\mathrm{Pb})$, mercury $(\mathrm{Hg})$, and cadmium $(\mathrm{Cd})$ (Kennelly, 2018). Various diseases have been linked to metal toxicity including softening of bones, renal dysfunction, neurological toxicity, hematological disorders, and skin diseases such as pigmentation, keratosis, leukomelanosis, and cancer (Chowdhury et al., 2016; Kim et al., 2019).

Naturally, heavy metals are released into environment through anthropogenic processes. There are different potential sources for drinking water contamination with heavy metals, most of which stem from urbanization and agricultural activities (Martín et al., 2015). As a result of urbanization, metals can be leached from pipes, storage tanks, coolers, and other constructions of water distribution system (Alabdula'aly and Khan, 2009). Moreover, industrial wastes, especially electronic ones, also contribute to the presence of heavy metals in different water sources (Chowdhury et al., 2016). The sediments below water column in aquatic bodies are well-known to accumulate heavy metals that may be re-suspended in the water (Wang et al., 2015; Wu et al., 2014). Besides the natural sources of ground water contamination, water is also prone to heavy metal contamination during consumption. Indeed, corrosion of pipe systems, coolers, and storage tanks is a significant source of $\mathrm{Cu}, \mathrm{Pb}, \mathrm{Ni}, \mathrm{Cr}, \mathrm{Fe}$, and $\mathrm{Zn}$ (Chowdhury et al., 2016).

Heavy metal contamination in drinking water has received much attention and various published reports have evaluated the concentrations of different elements in drinking water in different countries around the globe (Akoto and Adiyiah, 2007; BorteySam et al., 2015; Ghaderpoori et al., 2018; Mirzabeygi et al., 2017; Rasool et al., 2016). Low- and middle-income countries are facing the challenge of reducing heavy metal contents below the permissible limits for
Correspondence: Dhary Alewy Almashhadany, Department of Medical Lab Science, College of Science, Knowledge University, Erbil City, Kurdistan Region, Iraq Tel.: +9647733565479

E-mail: dhary.hammed@Knowledge.edu.krd

Key words: heavy metals; heavy metal pollution index; health risk.

Conflict of interests: The authors declare no conflict of interest.

Funding: This study was supported by Knowledge University and Salahaddin University- Erbil.

Availability of data and materials: All data available within the text.

Received for publication: 31 Januaruy 2020.

Revision received: 4 August 2020.

Accepted for publication: 7 August 2020 .

This work is licensed under a Creative Commons Attribution-NonCommercial 4.0 International License (CC BY-NC 4.0).

(C) Copyright: the Author(s), 2020

Licensee PAGEPress, Italy

Italian Journal of Food Safety 2020; 9:8862

doi:10.4081/ijfs.2020.8862

drinking water (Chowdhury et al., 2016). In Erbil city, the quality of drinking water supplied to school is derived from groundwater and rivers. To the best of our knowledge, there is no previous study assessed the levels of elements in schools drinking water tanks in Erbil city. Therefore, this study was conducted to assess, the water quality indices, multiple elements in drinking water, and accumulated tanks sediment collected from different schools in Erbil city, Kurdistan Region, Iraq.

\section{Materials and Methods}

\section{Chemicals}

All chemicals used in this work were extra pure including 50\% hydrogen peroxide $\left(\mathrm{H}_{2} \mathrm{O}_{2}\right), 37 \%$ hydrochloric acid $(\mathrm{HCl})$, and $65 \%$ nitric acid $\left(\mathrm{HNO}_{3}\right)$ purchased from Scharlau, Spain.

\section{Study area and samples collection}

Erbil is the capital and most populated city in the Kurdistan Region located at the north of Iraq $\left(36^{\circ} 11^{\prime} 28.28^{\prime \prime} \mathrm{N}, 44^{\circ} 0^{\prime} 33.08^{\prime \prime}\right.$ E). The total city area is about $115 \mathrm{~km}^{2}(44$ sq mi), and its inhabitants are about 879,000 people. 
A total of 18 basic schools offering primary and intermediate phases $\left(1^{\text {st }}-9^{\text {th }}\right.$ grades) in Erbil city (Figure 1) were selected for this study. Drinking water storage tanks were available in all targeted schools. The sampling process was done during MarchMay 2019. The details of targeted schools are summarized in Table 1.

Before the collection of the drinking water samples, the sampling containers were directly washed with the water of the sampled tank at a specific sampling site. Drinking water samples were acid-preserved immediately after collection. Measured 0.5 $\mathrm{mL}$ of dilute nitric acid $(1: 1 \mathrm{v} / \mathrm{v})$ was directly used as acid preservation and individually added to a $25 \mathrm{~mL}$ collected aliquot of drinking water. Drinking water samples and accumulated sediments from floors of storage tanks were separately collected, labelled, stored in a polypropylene bottle and transported to the laboratory for processing and chemical analysis.

\section{Preparation and digestion of the samples}

Prior to wet digestion process, all glassware items were rinsed with extra pure diluted nitric acid and then cleaned thoroughly with deionized water. The direct analyses for drinking water samples were performed according to previously published protocols of the United States Environmental Protection Agency (US EPA, 1992, 1994). Strong wet digestion was also applied to sediment samples (US EPA, 1996).
Collected sediment samples were firstly dried in an oven at $105{ }^{\circ} \mathrm{C}$ for $12 \mathrm{~h}$ till constant weights were obtained.

The dried sediment samples were crushed and mixed thoroughly to achieve homogeneity. One gram of dried sediment was placed into appropriate digestion conical flasks containing $10 \mathrm{~mL}(1: 1)$ of $65 \% \mathrm{HNO}_{3}$. The samples were left for 10 minutes for reflux and strong acid digestion in a classic digestion-heater. This step was repeated using $5 \mathrm{~mL}$ of concentrated $\mathrm{HNO}_{3}$. After cooling, $2 \mathrm{~mL}$ of distilled water and $3 \mathrm{~mL}$ of $\mathrm{H}_{2} \mathrm{O}_{2}(50 \%)$ were successively added to each digestion conical flask until bubbling subsides. The solution was refluxed and treated with $10 \mathrm{~mL}$ of concentrated $\mathrm{HCl}$ for 15 minutes to achieve complete digestion. Each solution was cooled at room temperature and transferred into $25 \mathrm{~mL}$ conical flask; the volume was diluted to the mark with deionized water. Finally, solutions were stored in appropriate plastic bottles for elemental analysis. All samples were prepared and analyzed in triplicate.

\section{Metal analysis}

Inductively Coupled Plasma Optical Emission Spectrometer (ICP-OES Spectro, Germany) was utilized for the elemental analysis. The levels of twenty-two chemical elements including, aluminum (Al), antimony (Sb), arsenic (As), barium (Ba), beryllium $(\mathrm{Be})$, cadmium $(\mathrm{Cd})$, calcium $(\mathrm{Ca})$, chromium $(\mathrm{Cr})$, cobalt $(\mathrm{Co})$, copper $(\mathrm{Cu})$, iron $(\mathrm{Fe})$, lead $(\mathrm{Pb})$, lithium $(\mathrm{Li})$, magnesium (Mg), manganese (Mn), molybdenum (Mo), nickel (Ni), potassium $(\mathrm{K})$, selenium $(\mathrm{Se})$, sodium $(\mathrm{Na})$, vanadium (V) and zinc ( $\mathrm{Zn}$ ) were investigated in all of the digested samples. Optimum operating conditions for the instrument were easily selected and conducted because all operating parameters are software controlled. The detailed fundamental features of the instrument, applied operating conditions in the analysis (Table 2), and selected wavelengths (lines) with a limit of detection (LOD) for the investigated metals (Tables 3 and 4) were chosen according to instruments manufacture (AMETEK, FHS22, 2015). The instrument was also calibrated against multielement standards, which were prepared from multi-elements stock solutions. The accuracy and precision of the method were investigated in excellent agreement for the analyzed elements using the standard reference material (NIST, SRM 1640a, Trace Elements in Natural Water). A LOD for the analyzed metals is calculated according to equation (1) as follows;

$$
\mathrm{LOD}=\frac{3 \mathrm{RSDb} \times \mathrm{c}}{\mathrm{SBR}}
$$

where, c, RSDb, and SBR denote concentration of the standard, relative standard deviation of 10 replicates of the blank, and signal to background ratio, respectively (AMETEK, FHS22, 2015).

The estimated concentrations were

Table 1. Information on the chosen schools.

\begin{tabular}{lcccc} 
School code & School name & Quarter/Location & \% students drinker & Storage tank status \\
S1 & Rozh & Karezan & 87 & Old \\
S2 & One of Shubat & Havalan & 76 & Old \\
\hline S3 & Darsem & Sheikh Ahmed & 59 & New \\
S4 & 14 Tamuz & Azadi & 37 & Large \& Old \\
\hline S5 & Shafaq & Kuran Makhmur & 68 & New \\
S6 & Aamad & Nawroz & 43 & Old \\
\hline S7 & Xabwr & Zamostiyan & New \\
S8 & Zanyari & Mamostiyan Zanko & 61 & Large \& Old \\
\hline S9 & Gardwn & Setaqan & 40 & Old \\
S10 & Hiwa & Kwestan & 67 & Large \& Old \\
\hline S11 & Leyla Zana & Salaheddin & 26 & New \\
S12 & Haval & Bakhtiari & 67 & New \\
\hline S13 & Bamok & Saidawa & 31 & Old \\
S14 & Makok & Shorsh & 76 & Old \\
\hline S15 & Krd Mandele & Tairawa & 72 & New \\
S16 & Snobar & Minaret & 24 & Old \\
\hline S17 & Xanzad & Kuran Ankawa & Old \\
S18 & Diyarbakir & 24 & \\
\hline
\end{tabular}


directly measured in $\mathrm{ng} \cdot \mathrm{mL}^{-1}$ (parts per billion) for both sediment and water samples. After using dilution factor, results were converted to $\mu \mathrm{g} \cdot \mathrm{mL}^{-1}$ (parts per million) for drinking water samples (Tables 5-7) and $\mu \mathrm{g} \cdot \mathrm{g}^{-1}$ (parts per million) for sediment samples (Tables 8 and 9). Metals were grouped into toxic and nontoxic categories based on medical perspective (Kennelly, 2018).

\section{Indexing approach}

In this study, two indices, namely, Heavy metal Evaluation Index (HEI) and Heavy metal Pollution Index (HPI) (Ghaderpoori et al., 2018), are used as quantitative assessment of drinking water quality in storage tanks of the selected schools in Erbil city. HEI for detected heavy metals in drinking water was calculated for each school according to the equation (2):

$$
\mathrm{HEI}=\sum_{i=1}^{n} \frac{H c}{H \max }
$$

where Hc is the detected value (and Hmax is the maximum permissible level of the ith parameter (metal). The classification of HEI is: low $(<10)$, medium $(10-20)$, and high (>20) (Ghaderpoori et al., 2018).

HPI which indicates the total quality of drinking water with respect to heavy metals (Mohan et al., 1996) was also calculated for

Table 2. Shows ICP operating conditions.

\begin{tabular}{lc} 
Parameters & Condition \\
Power & $1280 \mathrm{~W}$ \\
Coolant flow & $13 \mathrm{~L} / \mathrm{min}$ \\
\hline Auxiliary flow & $0.8 \mathrm{~L} / \mathrm{min}$ \\
Nebulizer flow & $0.75 \mathrm{~L} / \mathrm{min}$ \\
\hline Plasma Torch & Quartz, demountable, $1.8 \mathrm{~mm}$ injector tube \\
Spray Chamber & Cyclonic \\
\hline Nebulizer & Seaspray \\
Sample aspiration rate & $2.0 \mathrm{~mL} / \mathrm{min}$ \\
\hline Replicate read time & $48 \mathrm{sec}$ per replicate
\end{tabular}

each school according to equation (3) as follows (Ghaderpoori et al., 2018):

$$
\mathrm{HPI}=\frac{\sum_{i=1}^{n} W i . Q i}{\sum_{i=1}^{n} W i}
$$

The sub-index (Qi) of the parameter is also calculated according to equation (4) as

\begin{tabular}{|c|c|c|c|c|c|}
\hline $\begin{array}{l}\text { Elements } \\
\text { (Symbol) }\end{array}$ & $\begin{array}{l}\text { Wavelength } \\
\text { Line (nm) }\end{array}$ & $\begin{array}{l}\text { LOD 3o } \\
\left(\mu g . L^{-1}\right)\end{array}$ & $\begin{array}{l}\text { Elements } \\
\text { (Symbol) }\end{array}$ & $\begin{array}{l}\text { Wavelength } \\
\text { Line (nm) }\end{array}$ & $\begin{array}{l}\text { LOD 3o } \\
\left(\mu g . L^{-1}\right)\end{array}$ \\
\hline Aluminum (Al) & 167.078 & 0.07 & Lead $(\mathrm{Pb})$ & 220.351 & 3.44 \\
\hline Antimony (Sb) & 206.833 & 4.5 & Lithium (Li) & 670.784 & 1.3 \\
\hline Arsenic (As) & 189.042 & 3.1 & Magnesium (Mg) & 279.553 & 0.02 \\
\hline Barium (Ba) & 455.404 & 0.12 & Manganese (Mn) & 257.610 & 0.08 \\
\hline Beryllium (Be) & 313.042 & 0.06 & Molybdenum (Mo) & 202.030 & 0.9 \\
\hline Cadmium (Cd) & 214.438 & 0.22 & Nickel (Ni) & 231.604 & 0.95 \\
\hline Calcium (Ca) & 393.366 & 0.05 & Potassium (K) & 766.491 & 46 \\
\hline Chromium (Cr) & 267.716 & 0.67 & Selenium (Se) & 196.090 & 6.8 \\
\hline Cobalt (Co) & 228.615 & 0.654 & Sodium (Na) & 589.592 & 8.5 \\
\hline Copper $(\mathrm{Cu})$ & 324.754 & 1.1 & Vanadium (V) & 311.071 & 1.3 \\
\hline Iron $(\mathrm{Fe})$ & 259.941 & 0.4 & Zinc (Zn) & 213.856 & 0.2 \\
\hline
\end{tabular}

Table 3. Shows limits of detection (LOD) for the selected wavelengths (lines) for elements.

Table 4. Standard values and applied parameters ( $\left.\mu \mathrm{g} . \mathrm{L}^{-1}\right)$ used for calculation of HEI and HPI according to WHO guidelines

\begin{tabular}{|c|c|c|c|c|c|}
\hline Heavy metal & MCL & Wi & li & Si & Hmax \\
\hline Arsenic (As) & 50 & 0.02 & 10 & 50 & 50 \\
\hline Cadmium (Cd) & 3 & 0.3 & 3 & 5 & 3 \\
\hline Chromium (Cr) & 50 & 0.02 & 50 & 1 & 50 \\
\hline Copper (Cu) & 1000 & 0.001 & 2000 & 1000 & 1000 \\
\hline Iron (Fe) & 200 & 0.005 & 200 & 300 & 200 \\
\hline Lead $(\mathrm{Pb})$ & 1.5 & 0.7 & 10 & 100 & 1.5 \\
\hline Manganese (Mn) & 50 & 0.02 & 500 & 100 & 50 \\
\hline Nickel (Ni) & 20 & 0.05 & 20 & 70 & 20 \\
\hline Zinc (Zn) & 5000 & 0.0002 & 3000 & 5000 & 5000 \\
\hline
\end{tabular}
(Ghaderpoori et al., 2018).

MAC, maximum admissible concentration/ upper permissible; W, the weightage was taken as the inverse of MAC (1/MAC); I, ideal value (highest permissible) in ppb; S, standard value (standard permissible) in ppb; Hmax, is the maximum permissible level; i, the guide value for the considered parameter (metal). The used value of constants and parameter terms must have the same unit in computing the HPI and HEI values. 
follows:

$Q i=\sum_{i=1}^{n} \frac{\{M i(-) I i\}}{(S i-I i)} \times 100$ where, Wi, Qi, and $\mathrm{n}$ denote the unit weightage of the ith parameter, the sub-index of the ith parameter, and the number of parameters considered, respectively. In addition, $\mathrm{Mi}, \mathrm{Si}$, and $\mathrm{Ii}$ are the detected value of heavy metal, standard value of the ith parameter, and ideal value of the ith parameter, respectively (Table 4). The negative sign (-) signifies numerical difference of the two values, ignoring the algebraic sign.

Low $(<100)$, the threshold risk $(=100)$, and high $(>100)$ are three categories that were used to classify the water quality based

Table 5. Concentrations $\left(\mu \mathrm{g} \cdot \mathrm{mL}^{-1}\right)$ of non-toxic metals in drinking water samples from the sampled schools $(\mathrm{n}=18)$.

\begin{tabular}{|c|c|c|c|c|c|c|c|c|c|}
\hline School code & Al & $\mathrm{Ca}$ & $\mathrm{Fe}$ & Li & $\mathrm{Mg}$ & Mn & Mo & K & $\mathrm{Na}$ \\
\hline S1 & $0.096 \pm 0.032$ & $10.23 \pm 0.61$ & $0.052 \pm 0.006$ & $0.005 \pm 0.001$ & $2.95 \pm 0.12$ & $0.002 \pm 0.001$ & BDL & $0.325 \pm 0.019$ & $3.95 \pm 0.17$ \\
\hline S2 & BDL & $10.41 \pm 0.44$ & $0.01 \pm 0.002$ & $0.005 \pm 0.003$ & $2.83 \pm 0.07$ & $0.001 \pm 0.001$ & BDL & $0.275 \pm 0.009$ & $3.86 \pm 0.09$ \\
\hline S3 & $0.005 \pm 0.009$ & $10.85 \pm 0.57$ & $0.009 \pm 0.002$ & $0.005 \pm 0.001$ & $3.21 \pm 0.20$ & $0.006 \pm 0.001$ & BDL & $0.267 \pm 0.013$ & $3.89 \pm 0.09$ \\
\hline S4 & BDL & $8.98 \pm 0.27$ & $0.003 \pm 0.002$ & $0.008 \pm 0.002$ & $4.65 \pm 0.12$ & BDL & BDL & $0.233 \pm 0.016$ & $13.65 \pm 0.13$ \\
\hline S5 & BDL & $10.27 \pm 0.97$ & $0.011 \pm 0.007$ & $0.005 \pm 0.002$ & $2.95 \pm 0.28$ & $0.001 \pm 0.001$ & BDL & $0.315 \pm 0.028$ & $4.51 \pm 0.14$ \\
\hline S6 & $0.045 \pm 0.013$ & $11.26 \pm 0.57$ & $0.023 \pm 0.002$ & $0.005 \pm 0.001$ & $3.29 \pm 0.21$ & $0.001 \pm 0.001$ & $0.43 \pm 0.001$ & $0.308 \pm 0.01$ & $4.58 \pm 0.11$ \\
\hline S7 & $0.007 \pm 0.012$ & $11.48 \pm 0.57$ & $0.02 \pm 0.009$ & $0.005 \pm 0.001$ & $3.28 \pm 0.16$ & $0.001 \pm 0.001$ & BDL & $0.271 \pm 0.019$ & $4.15 \pm 0.04$ \\
\hline S8 & $0.004 \pm 0.004$ & $11.75 \pm 0.19$ & $0.017 \pm 0.003$ & $0.008 \pm 0.001$ & $3.57 \pm 0.25$ & $0.001 \pm 0.001$ & BDL & $0.311 \pm 0.03$ & $5.03 \pm 0.20$ \\
\hline S9 & BDL & $11.31 \pm 0.43$ & $0.013 \pm 0.006$ & $0.006 \pm 0.001$ & $3.23 \pm 0.16$ & $0.001 \pm 0.001$ & BDL & $0.283 \pm 0.009$ & $4.05 \pm 0.11$ \\
\hline S10 & BDL & $11.18 \pm 0.70$ & $0.009 \pm 0.005$ & $0.004 \pm 0.002$ & $3.24 \pm 0.19$ & BDL & $0.001 \pm 0.001$ & $0.274 \pm 0.008$ & $4.08 \pm 0.07$ \\
\hline S11 & BDL & $15.17 \pm 1.94$ & $0.02 \pm 0.015$ & $0.01 \pm 0.001$ & $5.69 \pm 0.74$ & BDL & BDL & $0.298 \pm 0.047$ & $7.81 \pm 0.29$ \\
\hline S12 & BDL & $10.94 \pm 0.52$ & $0.02 \pm 0.022$ & $0.007 \pm 0.001$ & $3.21 \pm 0.16$ & $0.001 \pm 0.001$ & BDL & $0.316 \pm 0.012$ & $4.26 \pm 0.08$ \\
\hline $\mathrm{S} 13$ & $0.096 \pm 0.015$ & $11.56 \pm 1.1$ & $0.034 \pm 0.003$ & $0.005 \pm 0.002$ & $3.85 \pm 0.50$ & $0.001 \pm 0.001$ & $0.001 \pm 0.001$ & $0.298 \pm 0.013$ & $6.21 \pm 0.35$ \\
\hline S14 & BDL & $13.99 \pm 0.92$ & $0.008 \pm 0.007$ & $0.009 \pm 0.001$ & $5.7 \pm 0.42$ & BDL & BDL & $0.259 \pm 0.037$ & $8.46 \pm 0.56$ \\
\hline S15 & $0.151 \pm 0.032$ & $10.68 \pm 0.82$ & $0.069 \pm 0.003$ & $0.01 \pm 0.002$ & $3.68 \pm 0.43$ & $0.003 \pm 0.001$ & $0.001 \pm 0.001$ & $0.348 \pm 0.024$ & $8.85 \pm 0.25$ \\
\hline S16 & BDL & $10.11 \pm 0.17$ & $0.007 \pm 0.002$ & $0.008 \pm 0.002$ & $3.2 \pm 0.06$ & BDL & $0.001 \pm 0.001$ & $0.35 \pm 0.001$ & $4.15 \pm 0.10$ \\
\hline S17 & $0.043 \pm 0.039$ & $14.34 \pm 1.07$ & $0.037 \pm 0.003$ & $0.01 \pm 0.002$ & $3.72 \pm 0.41$ & $0.008 \pm 0.001$ & $0.001 \pm 0.001$ & $0.403 \pm 0.028$ & $6.65 \pm 0.18$ \\
\hline S18 & BDL & $9.59 \pm 0.53$ & $0.002 \pm 0.001$ & $0.013 \pm 0.002$ & $5.95 \pm 0.32$ & BDL & BDL & $0.428 \pm 0.015$ & $20.16 \pm 0.48$ \\
\hline Mean & 0.025 & 11.339 & 0.020 & 0.007 & 3.789 & 0.002 & 0.024 & 0.309 & 6.572 \\
\hline WHO PL & 0.150 & NS & 2.000 & NS & NS & 0.400 & 0.070 & NS & NS \\
\hline
\end{tabular}

BDL: below the method detection limit, PL: permissible limit, NS: Not specified, WHO: World Health Organization.

Table 6. concentrations $\left(\mu \mathrm{g} \mathrm{mL} \mathrm{m}^{-1}\right)$ of toxic metals in drinking water samples from the sampled schools $(\mathrm{n}=18)$.

\begin{tabular}{|c|c|c|c|c|c|c|}
\hline School code & $\mathrm{Ba}$ & Co & $\mathrm{Ni}$ & Se & V & $\mathrm{Zn}$ \\
\hline S1 & $0.034 \pm 0.001$ & BDL & BDL & $0.029 \pm 0.004$ & $0.001 \pm 0.001$ & $0.042 \pm 0.003$ \\
\hline S2 & $0.027 \pm 0.001$ & $0.001 \pm 0.001$ & $0.002 \pm 0.001$ & $0.016 \pm 0.01$ & $0.002 \pm 0.001$ & $0.016 \pm 0.001$ \\
\hline S3 & $0.029 \pm 0.001$ & $0.002 \pm 0.001$ & $0.002 \pm 0.002$ & $0.01 \pm 0.013$ & $0.002 \pm 0.001$ & BDL \\
\hline S4 & $0.086 \pm 0.001$ & $0.001 \pm 0.001$ & BDL & $0.005 \pm 0.001$ & $0.002 \pm 0.001$ & $0.121 \pm 0.008$ \\
\hline S5 & $0.049 \pm 0.003$ & $0.002 \pm 0.001$ & BDL & $0.022 \pm 0.001$ & $0.001 \pm 0.001$ & $0.005 \pm 0.001$ \\
\hline S6 & $0.034 \pm 0.002$ & $0.001 \pm 0.001$ & $0.003 \pm 0.001$ & $0.022 \pm 0.017$ & $0.001 \pm 0.001$ & $0.003 \pm 0.001$ \\
\hline S7 & $0.033 \pm 0.001$ & BDL & $0.001 \pm 0.001$ & $0.015 \pm 0.012$ & $0.002 \pm 0.001$ & $0.004 \pm 0.001$ \\
\hline S8 & $0.041 \pm 0.003$ & $0.001 \pm 0.001$ & BDL & $0.014 \pm 0.009$ & $0.001 \pm 0.001$ & BDL \\
\hline S9 & $0.031 \pm 0.002$ & $0.001 \pm 0.001$ & $0.001 \pm 0.001$ & $0.006 \pm 0.008$ & $0.002 \pm 0.001$ & $0.006 \pm 0.001$ \\
\hline $\mathrm{S} 10$ & $0.030 \pm 0.001$ & $0.001 \pm 0.001$ & $0.001 \pm 0.001$ & $0.01 \pm 0.012$ & $0.002 \pm 0.001$ & BDL \\
\hline S11 & $0.093 \pm 0.006$ & $0.001 \pm 0.001$ & $0.001 \pm 0.001$ & $0.029 \pm 0.012$ & $0.001 \pm 0.001$ & $0.012 \pm 0.004$ \\
\hline S12 & $0.033 \pm 0.001$ & $0.002 \pm 0.001$ & $0.001 \pm 0.001$ & $0.022 \pm 0.006$ & $0.002 \pm 0.001$ & $0.002 \pm 0.001$ \\
\hline S13 & $0.034 \pm 0.002$ & $0.001 \pm 0.001$ & $0.002 \pm 0.001$ & $0.019 \pm 0.005$ & $0.001 \pm 0.001$ & BDL \\
\hline S14 & $0.104 \pm 0.007$ & $0.001 \pm 0.001$ & BDL & $0.016 \pm 0.002$ & $0.001 \pm 0.001$ & $0.001 \pm 0.001$ \\
\hline S15 & $0.048 \pm 0.001$ & $0.001 \pm 0.001$ & $0.003 \pm 0.001$ & $0.02 \pm 0.002$ & $0.002 \pm 0.001$ & $0.055 \pm 0.0017$ \\
\hline S16 & $0.029 \pm 0.001$ & $0.001 \pm 0.001$ & $0.006 \pm 0.001$ & $0.022 \pm 0.013$ & $0.001 \pm 0.001$ & $0.008 \pm 0.001$ \\
\hline S17 & $0.041 \pm 0.001$ & $0.001 \pm 0.001$ & $0.002 \pm 0.003$ & $0.018 \pm 0.005$ & $0.001 \pm 0.001$ & $0.063 \pm 0.006$ \\
\hline S18 & $0.056 \pm 0.001$ & BDL & $0.006 \pm 0.001$ & $0.007 \pm 0.001$ & $0.002 \pm 0.001$ & $0.017 \pm 0.002$ \\
\hline Mean & 0.046 & 0.001 & 0.002 & 0.017 & 0.002 & 0.020 \\
\hline WHO PL & 0.7 & NS & 0.07 & 0.04 & NS & NS \\
\hline
\end{tabular}

BDL: below method detection limit, PL: permissible limit, NS: Not specified. 
on HPI value. It verifies that 100 is selected as the critical pollution index of HPI value for drinking water. Water is not suitable for drinking when HPI is greater than 100 value (Ghaderpoori et al., 2018). For the present study, nine heavy metals including As, Cd, $\mathrm{Cr}, \mathrm{Cu}, \mathrm{Fe}, \mathrm{Pb}, \mathrm{Mn}, \mathrm{Ni}$, and $\mathrm{Zn}$ were used in computing the HPI and HEI parameters.

\section{Statistical analysis}

All data were analyzed in Microsoft Excel 2016 and version 25 of SPSS (IBM, Chicago, USA). Due to non-normal distribution of values of metals concentrations, nonparametric analyses were employed. Mann-Whitney $U$ test was used to analyze the difference between the concentrations of metals in drinking water and sediments at significance level of 0.05 .

\section{Results and Discussion}

A total of 18 schools in Erbil city were included in this study for evaluation of 22 metal elements in drinking water and sediments accumulated at the floor of water storage tanks. The average load of targeted elements in sediments was very high and equal to $71.64 \pm 15.23 \times 10^{3} \mu \mathrm{g} . \mathrm{g}^{-1}$, while the load in drinking water was $22.17 \pm 2.79$ $\mu \mathrm{g} . \mathrm{mL}^{-1}$. Detail loads in water and sediments, which represent the summation calculation of the means level of all detected metals for each of the analyzed samples, are depicted in Figures 2 and 3. A weak negative correlation was found between load of elements in drinking water and sediments in the same tank $(\mathrm{r}=-0.220)$.

\section{Metal concentration in water}

The results of analysis nontoxic metals in drinking water are shown in Table 5. The average concentration of metals followed the decreasing order of: $\mathrm{Ca}>\mathrm{Na}>\mathrm{Mg}>\mathrm{K}>\mathrm{Al}$ $>\mathrm{Mo}>\mathrm{Fe}>\mathrm{Li}>\mathrm{Mn}$. Only water of Aamad and Krd Mandele schools exceeded the WHO permissible limits for molybdenum and aluminum, respectively (Table 5). The concentrations of toxic metals are summarized in Table 6. No water sample from the schools exceeded the WHO permissible limits for all tested toxic metals. These findings are consistent with studies conducted in Jordan (Alomary, 2013). Strikingly, antimony (Sb), arsenic (As), beryllium $(\mathrm{Be})$, cadmium $(\mathrm{Cd})$, chromium $(\mathrm{Cr})$, copper $(\mathrm{Cu})$ and Lead $(\mathrm{Pb})$ were not

Table 7. Calculated HPI and HEI for heavy metals in drinking water from the sampled schools.

\begin{tabular}{|c|c|c|c|c|c|c|c|c|c|}
\hline School code & HPI & HPI classify & HEI & HEI classify & School code & HPI & HPI classify & HEI & HEI classify \\
\hline S1 & 54.4510 & LHM & 0.3084 & LHM & $\mathrm{S} 10$ & 54.5633 & LHM & 0.0950 & LHM \\
\hline S2 & 54.4647 & LHM & 0.1732 & LHM & S11 & 54.5139 & LHM & 0.1524 & LHM \\
\hline S3 & 54.4469 & LHM & 0.2650 & LHM & S12 & 54.5096 & LHM & 0.1704 & LHM \\
\hline S4 & 54.6787 & LHM & 0.0392 & LHM & S13 & 54.3573 & LHM & 0.2900 & LHM \\
\hline S5 & 54.6394 & LHM & 0.0760 & LHM & S14 & 54.6574 & LHM & 0.0402 & LHM \\
\hline S6 & 54.3169 & LHM & 0.2856 & LHM & S15 & 54.1015 & LHM & 0.5660 & LHM \\
\hline S7 & 54.5095 & LHM & 0.1708 & LHM & S16 & 54.1243 & LHM & 0.3366 & LHM \\
\hline S8 & 54.6126 & LHM & 0.1050 & LHM & S17 & 54.3119 & LHM & 0.4576 & LHM \\
\hline \multirow[t]{2}{*}{ S9 } & 54.5409 & LHM & 0.1362 & LHM & S18 & 54.1466 & LHM & 0.3134 & LHM \\
\hline & & & & & Average & 54.442 & LHM & 0.2212 & LHM \\
\hline
\end{tabular}

LHM; Low Heavy Metals.

Table 8. Concentrations $\left(\mu \mathrm{g} \mathrm{g}^{-1}\right)$ of non-toxic metals in sediments samples from sampled schools $(\mathrm{n}=18)$.

\begin{tabular}{|c|c|c|c|c|c|c|c|c|c|}
\hline \multicolumn{2}{|c|}{ School code Al } & $\mathrm{Ca}$ & $\mathrm{Fe}$ & Li & $\mathrm{Mg}$ & Mn & Mo & K & $\mathrm{Na}$ \\
\hline S1 & $11178 \pm 2089$ & $60137 \pm 219$ & $10941 \pm 319$ & $17.2 \pm 0.62$ & $10839 \pm 48$ & $321.26 \pm 17.66$ & $0.597 \pm 0.584$ & $271.08 \pm 25.40$ & $140.27 \pm 41.27$ \\
\hline S2 & $13457 \pm 1634$ & $60176 \pm 291$ & $24278 \pm 4375$ & $18.45 \pm 1.13$ & $7856 \pm 301$ & $231.22 \pm 55.86$ & $0.912 \pm 0.412$ & $242.56 \pm 22.20$ & $110.16 \pm 9.19$ \\
\hline S3 & $5437 \pm 269$ & $60485 \pm 85$ & $1399 \pm 30$ & $8.44 \pm 0.58$ & $2619 \pm 53$ & $47.16 \pm 0.44$ & BDL & $142.28 \pm 26.27$ & $127.17 \pm 57.58$ \\
\hline S4 & $10283 \pm 472$ & $28379 \pm 132$ & $12042 \pm 1356$ & $14.58 \pm 0.97$ & $2855 \pm 226$ & $160.7 \pm 29.95$ & $0.63 \pm 0.124$ & $466.05 \pm 43.77$ & $146.0 \pm 7.22$ \\
\hline S5 & $281.3 \pm 11$ & $60361 \pm 20$ & $521 \pm 61$ & $0.671 \pm 0.095$ & $2077 \pm 62$ & $6.06 \pm 0.36$ & BDL & $58.86 \pm 5.43$ & $121.87 \pm 64.65$ \\
\hline S6 & $2031 \pm 85$ & $60374 \pm 48$ & $1411 \pm 105$ & $5.19 \pm 0.17$ & $1868 \pm 105$ & $15.89 \pm 0.68$ & BDL & $114.91 \pm 13.07$ & $139.24 \pm 74.87$ \\
\hline S7 & $1337 \pm 74$ & $60071 \pm 266$ & $6481 \pm 473$ & $3.42 \pm 0.17$ & $1417 \pm 41$ & $15.02 \pm 1.09$ & $0.602 \pm 0.064$ & $102.85 \pm 1.13$ & $143.56 \pm 16.64$ \\
\hline S8 & $1402 \pm 200$ & $60317 \pm 54$ & $1762 \pm 9$ & $3.43 \pm 0.37$ & $1137 \pm 42$ & $16.48 \pm 2.66$ & BDL & $109.06 \pm 8.93$ & $130.08 \pm 30.72$ \\
\hline \$9 & $3446 \pm 514$ & $3921 \pm 515$ & $40523 \pm 27$ & $6.19 \pm 0.92$ & $695 \pm 77$ & $438.97 \pm 6.29$ & $5.86 \pm 0.20$ & $183.04 \pm 15.78$ & $236.88 \pm 18.62$ \\
\hline S10 & $372.7 \pm 46$ & $60356 \pm 55$ & $1680 \pm 135$ & $1.00 \pm 0.03$ & $1366 \pm 138$ & $4.72 \pm 1.78$ & BDL & $39.95 \pm 11.73$ & $74.77 \pm 18.42$ \\
\hline S11 & $1681 \pm 78$ & $54767 \pm 961$ & $15624 \pm 589$ & $2.41 \pm 0.08$ & $423 \pm 22$ & $92.58 \pm 11.15$ & $1.6 \pm 0.16$ & $127.53 \pm 43.18$ & $117.88 \pm 50.87$ \\
\hline S12 & $4005 \pm 233$ & $60171 \pm 183$ & $1942 \pm 163$ & $12.44 \pm 0.35$ & $1718 \pm 102$ & $29.02 \pm 2.22$ & BDL & $203.96 \pm 6.07$ & $69.85 \pm 10.56$ \\
\hline $\mathrm{S} 13$ & $2068 \pm 142$ & $60321 \pm 219$ & $452 \pm 57$ & $4.77 \pm 0.19$ & $1406 \pm 23$ & $13.05 \pm 1.24$ & BDL & $126.53 \pm 8.71$ & $127.45 \pm 10.11$ \\
\hline S14 & $621 \pm 100$ & $59930 \pm 330$ & $8545 \pm 42$ & $1.17 \pm 0.13$ & $189 \pm 15$ & $27.84 \pm 11.48$ & $0.506 \pm 0.104$ & $76.94 \pm 10.58$ & $110.06 \pm 3.22$ \\
\hline S15 & $2325 \pm 57$ & $60114 \pm 271$ & $1837 \pm 116$ & $4.81 \pm 0.06$ & $1246 \pm 51$ & $22.01 \pm 1.49$ & BDL & $165.54 \pm 10.90$ & $78.13 \pm 14.0$ \\
\hline S16 & $2741 \pm 154$ & $60168 \pm 281$ & $1278 \pm 91$ & $7.37 \pm 0.24$ & $1416 \pm 81$ & $22.35 \pm 1.32$ & $\mathrm{BDL}$ & $49.33 \pm 0.82$ & $254.73 \pm 7.16$ \\
\hline S17 & $5044 \pm 463$ & $60026 \pm 359$ & $5324 \pm 701$ & $9.64 \pm 0.7$ & $1553 \pm 122$ & $44.86 \pm 4.35$ & $0.294 \pm 0.051$ & $266.90 \pm 21.59$ & $109.49 \pm 24.27$ \\
\hline S18 & $312.8 \pm 29$ & $60297 \pm 74$ & $264 \pm 229$ & $0.769 \pm 0.069$ & $99.00 \pm 6.0$ & $2.73 \pm 0.67$ & BDL & $166.32 \pm 4.96$ & $89.26 \pm 0.02$ \\
\hline Mean & 3779.04 & 54770.6 & 7567.44 & 6.78 & 2265.5 & 93.94 & 0.61 & 161.87 & 129.27 \\
\hline
\end{tabular}

BDL: below method detection limit. 
detected in any water sample.

In this study, the data of schools drinking water have been mainly used for the calculation of Heavy-metal Pollution Index and Heavy-metal Evaluation Index. Measured values of HEI and HPI for drinking water samples are presented in Table 7. As shown in the table, the calculated HPI values for all samples were in the suitable range for drinking purposes as they below the critical threshold value of 100 . The results revealed that the average values of HPI and HEI in the water samples were 54.442 and 0.221 (Table 7), respectively, and this indicates low contamination levels in terms of heavy metals.

The recorded results in this study were in a good agreement with several previous published studies in this city. Issa and Alrwai (2018) stated that the quality of the drinking water supply for Three Water Treatment Plants of Erbil City never reached the level of marginal or poor over the time investigated. Kafia et al., (2009) revealed that most of the parameters analyzed for drinking water from Water Treatment Plants on Greater Zab River in Erbil city were within the guidelines given by WHO for drinking water purposes after applying usual treatments.

Several recently published studies were conducted for the assessment of drinking water quality around the Erbil city location such as Halabja city (Salih et al., 2015), Zakho city (Salim et al., 2017), Garmian city
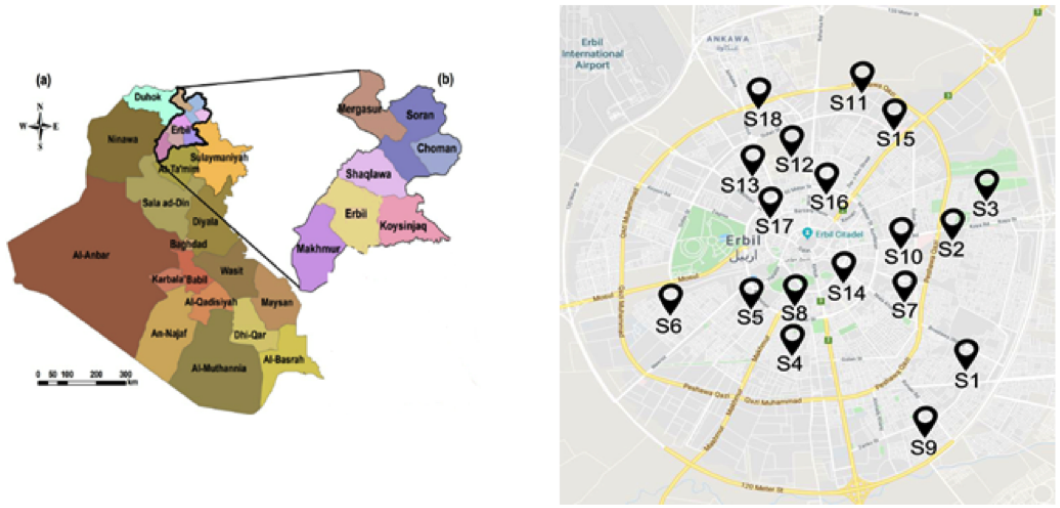

Figure 1. Map of study area and sampled schools, (a) Iraq, (b) Erbil city in Kurdistan Region of Iraq, and (c) schools' locations (S1-S18) in the study area inside Erbil city center.

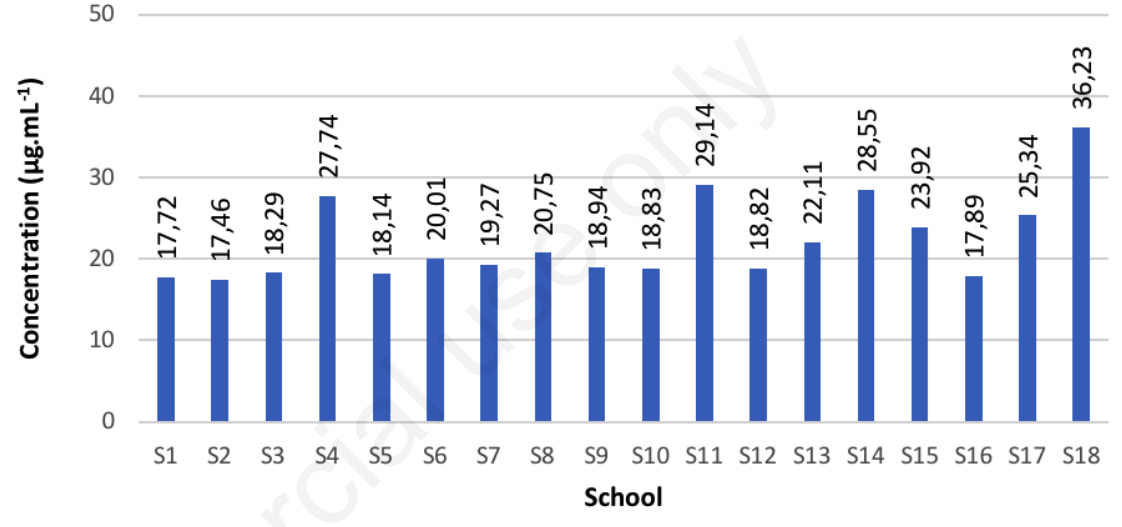

Figure 2. Total load of elements in drinking water samples.

Table 9. Concentrations ( $\mu \mathrm{g} \cdot \mathrm{g}-1)$ of toxic metals in sediments samples from chosen schools in Erbil city ( $\mathrm{n}=18)$.

\begin{tabular}{|c|c|c|c|c|c|c|c|c|c|c|c|c|c|}
\hline $\begin{array}{l}\text { School } \\
\text { code }\end{array}$ & As & $\mathrm{Ba}$ & $\mathrm{Be}$ & $\mathrm{Cd}$ & $\mathrm{Cr}$ & $\mathrm{Co}_{0}$ & $\mathrm{Cu}$ & $\mathrm{Ni}$ & $\mathrm{Pb}$ & $\mathrm{Sb}$ & Se & V & $\mathrm{Zn}$ \\
\hline S1 & $5.35 \pm 0.79$ & $94.03 \pm 10.62$ & $0.224 \pm 0.059$ & $1.34 \pm 0.53$ & $31.17 \pm 4.83$ & $7.52 \pm 0.39$ & $25.97 \pm 1.31$ & $52.98 \pm 7.04$ & $30.45 \pm 5.77$ & BDL & $3.799 \pm 0.89$ & $13.08 \pm 0.84$ & $12905 \pm 1018$ \\
\hline \$2 & $5.78 \pm 1.52$ & $98.8 \pm 6.35$ & $0.168 \pm 0.058$ & $1.06 \pm 0.28$ & $22.65 \pm 5.66$ & $5.55 \pm 1.01$ & $16.94 \pm 4.62$ & $45.72 \pm 7.86$ & $22.55 \pm 4.38$ & BDL & $3.522 \pm 0.47$ & $10.41 \pm 1.59$ & $6596 \pm 1423$ \\
\hline S3 & $1.52 \pm 0.29$ & $51.39 \pm 4.78$ & $0.026 \pm 0.002$ & $0.286 \pm 0.015$ & $6.99 \pm 1.33$ & $1.27 \pm 0.03$ & $3.83 \pm 0.19$ & $14.86 \pm 0.51$ & $2.89 \pm 0.25$ & BDL & $1.408 \pm 0.29$ & $2.19 \pm 0.03$ & $525 \pm 21$ \\
\hline S4 & $2.31 \pm 0.14$ & $195.2 \pm 12.2$ & $0.199 \pm 0.01$ & $0.933 \pm 0.103$ & $12.3 \pm 0.86$ & $2.57 \pm 0.28$ & $12.82 \pm 0.54$ & $16.8 \pm 1.20$ & $70.81 \pm 4.83$ & BDL & $1.236 \pm 0.75$ & $11.85 \pm 0.56$ & $9607 \pm 269$ \\
\hline S5 & $0.638 \pm 0.324$ & $60.83 \pm 0.97$ & BDL & $0.036 \pm 0.005$ & $0.605 \pm 0.124$ & $0.072 \pm 0.05$ & $0.817 \pm 0.29$ & $2.41 \pm 0.17$ & BDL & BDL & $1.608 \pm 0.40$ & $0.428 \pm 0.03$ & $123 \pm 5$ \\
\hline S6 & $1.16 \pm 0.20$ & $38.53 \pm 1.34$ & $0.001 \pm 0.001$ & $0.092 \pm 0.03$ & $1.61 \pm 0.07$ & $0.422 \pm 0.006$ & $0.658 \pm 0.07$ & $4.54 \pm 0.31$ & $0.619 \pm 0.21$ & BDL & $0.70 \pm 0.26$ & $1.12 \pm 0.001$ & $124 \pm 13$ \\
\hline S7 & BDL & $37.26 \pm 3.49$ & BDL & $0.228 \pm 0.04$ & $1.11 \pm 0.38$ & $0.506 \pm 0.047$ & $2.14 \pm 0.26$ & $10.66 \pm 0.63$ & $2.44 \pm 0.02$ & $1.6 \pm 0.48$ & $0.134 \pm 0.23$ & $2.77 \pm 0.16$ & $251 \pm 13$ \\
\hline S8 & $0.89 \pm 0.236$ & $37.37 \pm 7.34$ & BDL & $0.12 \pm 0.015$ & $1.17 \pm 0.17$ & $0.342 \pm 0.072$ & $1.73 \pm 0.19$ & $6.0 \pm 0.71$ & $2.34 \pm 0.51$ & $0.045 \pm 0.001$ & $0.686 \pm 0.25$ & $1.35 \pm 0.17$ & $334 \pm 29$ \\
\hline$\$ 9$ & $14.05 \pm 1.97$ & BDL & BDL & $1.35 \pm 0.04$ & $10.55 \pm 0.78$ & $9.06 \pm 0.23$ & $42.82 \pm 0.76$ & $66.71 \pm 1.31$ & $17.29 \pm 1.07$ & $17.17 \pm 1.46$ & $3.024 \pm 5.24$ & $13.38 \pm 0.73$ & $2113 \pm 108$ \\
\hline$\$ 10$ & $0.841 \pm 0.31$ & $11.74 \pm 2.16$ & BDL & BDL & BDL & $0.132 \pm 0.061$ & $0.60 \pm 0.215$ & $1.5 \pm 0.08$ & BDL & BDL & $1.521 \pm 0.53$ & $0.456 \pm 0.13$ & $567 \pm 67$ \\
\hline S11 & $4.86 \pm 0.30$ & $180.4 \pm 7.28$ & $0.033 \pm 0.006$ & $0.831 \pm 0.03$ & $5.26 \pm 0.44$ & $2.43 \pm 0.23$ & $19.66 \pm 2.03$ & $18.62 \pm 1.43$ & $93.06 \pm 3.6$ & $\mathrm{BDL}$ & $0.099 \pm 0.17$ & $8.28 \pm 0.34$ & $5639 \pm 64$ \\
\hline$\$ 12$ & $0.956 \pm 0.188$ & $19.02 \pm 0.72$ & $0.033 \pm 0.002$ & $0.233 \pm 0.01$ & $3.77 \pm 0.21$ & $0.797 \pm 0.081$ & $2.50 \pm 0.24$ & $11.99 \pm 0.84$ & $3.46 \pm 0.16$ & $0.187 \pm 0.226$ & $1.764 \pm 0.44$ & $2.05 \pm 0.04$ & $229 \pm 7$ \\
\hline$\$ 13$ & $1.02 \pm 0.16$ & $10.13 \pm 2.68$ & $0.002 \pm 0.002$ & $0.082 \pm 0.01$ & $2.01 \pm 0.29$ & $0.308 \pm 0.027$ & $1.91 \pm 0.11$ & $5.03 \pm 0.42$ & $2.43 \pm 0.63$ & BDL & $1.63 \pm 0.27$ & $0.988 \pm 0.08$ & $508 \pm 43$ \\
\hline S14 & BDL & $298.7 \pm 9.57$ & BDL & $0.173 \pm 0.028$ & $1.62 \pm 0.31$ & $0.53 \pm 0.06$ & $2.86 \pm 0.95$ & $1.98 \pm 0.24$ & $18.31 \pm 0.26$ & BDL & BDL & $4.78 \pm 0.30$ & $3279 \pm 54$ \\
\hline S15 & $1.74 \pm 0.61$ & $7.97 \pm 2.75$ & $0.009 \pm 0.002$ & $0.071 \pm 0.007$ & $1.74 \pm 0.12$ & $0.449 \pm 0.044$ & $1.49 \pm 0.34$ & $5.08 \pm 0.21$ & $3.32 \pm 0.58$ & BDL & $1.183 \pm 0.71$ & $1.56 \pm 0.10$ & $2263 \pm 33$ \\
\hline S16 & $0.862 \pm 0.402$ & $7.11 \pm 2.53$ & BDL & $0.18 \pm 0.01$ & $2.49 \pm 0.21$ & $0.562 \pm 0.054$ & $1.68 \pm 0.18$ & $6.58 \pm 0.49$ & $2.02 \pm 0.25$ & BDL & $1.40 \pm 0.81$ & $1.6 \pm 0.07$ & $273 \pm 25$ \\
\hline S17 & $0.426 \pm 0.148$ & $90.38 \pm 11.09$ & $0.044 \pm 0.007$ & $0.359 \pm 0.063$ & $3.86 \pm 0.55$ & $1.29 \pm 0.19$ & $3.03 \pm 0.38$ & $10.17 \pm 1.06$ & $6.25 \pm 0.55$ & BDL & $1.125 \pm 0.43$ & $3.44 \pm 0.38$ & $3083 \pm 91$ \\
\hline$\$ 18$ & $0.571 \pm 0.255$ & $247.8 \pm 26.77$ & $0.014 \pm 0.002$ & BDL & $0.243 \pm 0.061$ & $0.108 \pm 0.019$ & $0.339 \pm 0.21$ & $0.32 \pm 0.09$ & BDL & BDL & $1.122 \pm 0.79$ & $0.509 \pm 0.03$ & $922 \pm 39$ \\
\hline Mean & 2.387 & 81.64 & 0.063 & 0.410 & 6.064 & 1.884 & 7.877 & 15.664 & 15.458 & 1.056 & 1.442 & 4.458 & 2741.72 \\
\hline
\end{tabular}




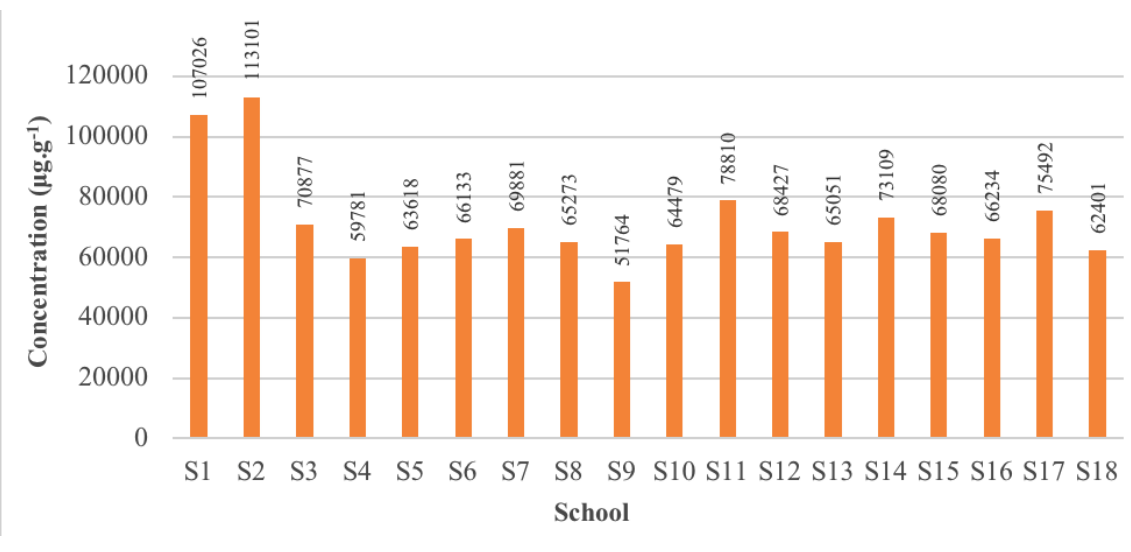

Figure 3. Total load of elements in sediments of drinking water tanks.

(Issa and Alshatteri, 2018), Duhok city (Meen et al., 2019), and Sulaimani city (Majid et al., 2019) from Kurdistan Region, Iraq. Meen et al., (2019) stated that results of HEI and HPI at all the studied sites in surface water of Duhok Dam were lower than permissible limits according to WHO standards. Nonetheless, the recorded results of HEI in drinking water of the Garmian city showed that $44 \%$ of the water samples are critically polluted in terms of heavy metals (Issa and Alshatteri, 2018). The concentrations of $\mathrm{Cd}$ and Ni metals in fifteen different well water in Zakho City were reported to exceed the maximum permissible limit set by the WHO standard (Salim et al., 2017).

\section{Metals concentration in sediments}

The results of nontoxic and toxic metals detected in storage tank sediments are shown in Tables 8 and 9. The average concentration of nontoxic metals followed the decreasing order of: $\mathrm{Ca}>\mathrm{Fe}>\mathrm{Al}>\mathrm{Mg}>\mathrm{K}>\mathrm{Na}>\mathrm{Mn}$ $>\mathrm{Li}>\mathrm{Mo}$. On the other hand, the average concentrations of toxic metals followed the decreasing order of: $\mathrm{Zn}>\mathrm{Ba}>\mathrm{Ni}>\mathrm{Pb}>\mathrm{Cu}$ $>\mathrm{Cr}>\mathrm{V}>\mathrm{As}>\mathrm{Co}>\mathrm{Se}>\mathrm{Sb}>\mathrm{Cd}>\mathrm{Be}$. Concentrations of metals in sediments were significantly higher than in drinking water of the same tank $(p>0.001)$. The elevated concentrations of metals in sediments may be attributed to corrosion of metal tanks, precipitation of particulate matter originally suspended in the water of river, and/or deposition of pollutants into the environment (Ziadat, 2005; Cobbina et al., 2015).

In Erbil city, drinking water sources are the Great Zab river, a tributary of Tigris river, and groundwater. In most cases, river water constitutes the majority of the daily supply of drinking water in most schools. Additionally, March-May period is within the rainy season of Erbil and deposition of flash floods and land runoff into the Great
Zab river contribute to increase of the particulate matter which precipitates in tanks after filling. The detected levels are alarming and deemed risky for human, especially children as a susceptible population. During daily refilling of tanks, sediments are resuspended in the water drunk by students.

Metal concentrations in tank of Rozh and One of Shubat schools were higher than other schools because of the fact that storage tanks were very old and lack proper covers and periodical maintenance. The quality of drinking water stored in tanks is affected by proper covering and the adopted sanitation practice (Hammad et al., 2008; Ziadat, 2005). Additionally, lack of proper cover of tanks was found to aid the atmospheric dissolution of heavy metals into the water (Al-Saleh and Al-Doush, 1998; Islam et al., 2014).

\section{Conclusions}

This study showed that multi-element levels of drinking water in basic schools in Erbil city (Iraq) were within the accepted limits specified by WHO. The results showed that, the average values of water quality indices (HEI \& HPI) were totally below the critical values and water samples have been identified suitable for drinking in terms of heavy metals. However, sediments of storage tanks in the schools were highly polluted with various toxic metals. These findings require urgent actions to mitigate pollution and prevent the transmission of toxic metals to students drinking from such tanks. Replacing the metal tanks with inert plastics ones and/or introduction of efficient filters may provide protection against metal sediments.

\section{References}

Akoto O, Adiyiah J, 2007. Chemical analysis of drinking water from some communities in the Brong Ahafo region. Int J Environ Sci Technol 4:211-4.

Al-Saleh I, Al-Doush I, 1998. Survey of trace elements in household and bottled drinking water samples collected in Riyadh, Saudi Arabia. Sci Total Environ 216:181-92.

Alabdula'aly AI, Khan MA, 2009. Heavy metals in cooler waters in Riyadh, Saudi Arabia. Environ Monitor Assess 157:238.

Ali H, Khan E, 2018. What are heavy metals? Long-standing controversy over the scientific use of the term 'heavy metals'-proposal of a comprehensive definition. Toxicol Environ Chem 100:6-19.

Alomary A, 2013. Determination of trace metals in drinking water in Irbid CityNorthern Jordan. Environ Monitor Assess 185:1969-75.

AMETEK, FHS22, 2015. "Spectro ICP Report; Analysis of Aqueous Solutions by ICP-OES with Radial Plasma Observation. Spectro Arcos, Spectro Analytical Instruments $\mathrm{GmbH}$ Germany" , Available from: https://extranet.spectro.com//media/31793ADA-B987-4D37-B597-0 4AFB66C7C22.pdf Accessed on July 10, 2020.

Baldwin A, Li B, Liu H, Mmereki, D, 2016. The Management of Hazardous Waste in Developing Countries. Chapter 3:39-50.

Bortey-Sam N, Nakayama SMM, Ikenaka Y, Akoto O, Baidoo E, Mizukawa $\mathrm{H}$, Ishizuka M. 2015. Health risk assessment of heavy metals and metalloid in drinking water from communities near gold mines in Tarkwa, Ghana. Environ Monitor Assess 187:112.

Chowdhury S, Mazumder MAJ, Al-Attas O, Husain T, 2016. Heavy metals in drinking water: Occurrences, implications, and future needs in developing countries. Sci Total Environ 569-570:476-88.

Cobbina SJ, Agoboh YP, Duwiejuah AB, Bakobie N, 2015. Evaluation of Stored Rainwater Quality in Basic Schools in the Tamale Metropolis, Ghana. Water Qual Expo Health 7:583-90.

Duffus JH, 2002. "Heavy metals" - A meaningless term? (IUPAC technical report). Pure Appl Chem 74:793-07.

Ferronato N, Torretta V, 2019. Waste mismanagement in developing countries: A review of global issues. Int J Environ Res Public Health 16:1-28.

Ghaderpoori M, Kamarehie B, Jafari A, Ghaderpoury A, Karami M, 2018. Heavy metals analysis and quality assessment in drinking water - Khorramabad city, 
Iran. Data Brief 16:685-92.

Hammad ZH, Ali AO, Ahmed HH, 2008. The quality of drinking water in storage tanks in Khartoum State. Kharoum Med J 1:78-80.

Islam MA, Karim MR, Higuchi T, Sakakibara H, Sekine M, 2014. Comparison of the trace metal concentration of drinking water supply options in southwest coastal areas of Bangladesh. Appl Water Sci 4:183-91.

Issa HM, Alrwai RA, 2018. Long-term Drinking Water Quality Assessment Using Index and Multivariate Statistical Analysis for Three Water Treatment Plants of Erbil City, Iraq. UKH J Sci Eng 2:39-48.

Issa HM, Alshatteri A, 2018. Assessment of Heavy Metals Contamination in Drinking Water of Garmian Region, Iraq. UHD J Sci Technol 2:40-53.

Kafia MS, Slaiman GM, Nazanin MS, 2009.

Physical and chemical status of drinking water from water treatment plants on Greater Zab River. J Appl Sci Environ Manag 13:89-92.

Kennelly P, 2018. The biochemical roles of transition metals. In: Rodwell V, Kennelly P, Bender P, Weil P, Botham K, eds. Harper's Illustrated Biochemistry. McGraw-Hill, New York, NY:92-102.

Kim JJ, Kim YS, Kumar V, 2019. Heavy metal toxicity: An update of chelating therapeutic strategies. J Trace Elements in Med Biol 54:226-31.

Majid SN, Khwakaram AI, Gado CS, Majeed BK, 2018. Pollution status evaluation of some heavy metals along some surface water sources by multivariate data analysis at Sulaimani governorate. J Zankoy Sulaimani 20:6380 .
Martín RJA, De Arana C, Ramos-Miras JJ, Gil C, Boluda R, 2015. Impact of 70 years urban growth associated with heavy metal pollution. Environ Pollut 196:156-63.

Meen HA, Rekani OA, Barwari VI, 2019. Application of Pollution indices for Heavy Metal Contamination Assessment in Surface Water of Duhok Dam (Kurdistan Region, Iraq). J Duhok Univ 22:252-64.

Mirzabeygi M, Abbasnia A, Yunesian M et al., 2017. Heavy metal contamination and health risk assessment in drinking water of Sistan and Baluchistan, Southeastern Iran. Human Ecol Risk Assess 23:1893-905.

Mohan SV, Nithila P, Reddy SJ, 1996. Estimation of heavy metals in drinking water and development of heavy metal pollution index. J Environ Sci Health Part A 31:283-9.

Rasool A, Farooqi A, Xiao T, Masood S, Kamran MA, bibi S, 2016. Elevated levels of arsenic and trace metals in drinking water of Tehsil Mailsi, Punjab, Pakistan. J Geochem Explor 169:89-99.

Salih FA, Kasem, AO, Abdullah TH, Othman NF, Mina MB, 2015. Assessment of the quality of drinking water of Halabja City-Iraqi Kurdistan. Kirkuk Univ J /Sci Studi (KUJSS) 10:259-72.

Salim HAM, Abdo HR, Buni FJ, Kareem IM, Najeeb MA, Hussen BA, 2017. Estimation and correlation analysis of heavy metals of some well water in Zakho City, Iraq. Sci J Univ Zakho 5:205-9.

United States Environmental Protection Agency, 1992. "Method 3005A: Acid Digestion of Waters for Total
Recoverable or Dissolved Metals for Analysis by Flame Atomic Absorption (FLAA) or Inductively Coupled Plasma (ICP) Spectroscopy" , Revision 1 Available from: https:/www.epa.gov/ hw-sw846/sw-846-test-method-3005aacid-digestion-waters-total-recoverableor-dissolved-metals Accessed on November 29,2019.

United States Environmental Protection Agency, 1994. "Method 200.7: Determination of Metals and Trace Elements in Water and Wastes by Inductively Coupled Plasma-Atomic Emission Spectrometry" Revision 4.4. Available from: https:/www.epa.gov/ sites/production/files/2015-08/documents/method_200-7_rev_4-4_1994.pdf . Accessed on November 29,2019 .

United States Environmental Protection Agency, 1996. "Method 3050B: Acid Digestion of Sediments, Sludges, and Soils," Revision 2. Washington, DC. Available from: https://www.epa.gov/ sites/production/files/2015-

06/documents/epa-3050b.pdf. Accessed on November 29,2019.

Wang G, A Y, Jiang H, Fu Q, Zheng B, 2015. Modeling the source contribution of heavy metals in surficial sediment and analysis of their historical changes in the vertical sediments of a drinking water reservoir. J Hydrol 520:37-51.

Wu B, Wang G, Wu J, Fu Q, Liu C, 2014. Sources of Heavy Metals in Surface Sediments and an Ecological Risk Assessment from Two Adjacent Plateau Reservoirs. PLoS ONE 9:e102101.

Ziadat AH, 2005. Impact of Storage Tanks on Drinking Water Quality in Al-Karak Province-Jordan. J Appl Sci 5:634-8. 